Research Paper

\title{
The C-reactive Protein/Albumin Ratio Is an independent Prognostic Factor for Overall Survival in Patients with Nasopharyngeal Carcinoma Receiving Intensity- Modulated Radiotherapy
}

\author{
Chang-Juan Tao*, Yuan-Yuan Chen*, Feng Jiang, Xing-Lai Feng, Qi-Feng Jin, Ting Jin, Yong-Feng Piao, \\ Xiao-Zhong Chen ${ }^{\otimes}$ \\ Department of Radiation Oncology, Zhejiang Provincial Cancer Hospital, Key Laboratory of Radiation Oncology of Zhejiang Province, Hangzhou, Zhejiang \\ Province, People's Republic of China \\ *These authors contributed equally to this work. \\ $\triangle$ Corresponding author: Xiao-Zhong Chen, Department of Radiation Oncology, Zhejiang Provincial Cancer Hospital, Key Laboratory of Radiation Oncology of \\ Zhejiang Province, Hangzhou, Zhejiang Province, People's Republic of China. Telephone: +86571-88122233; Fax: +86571-88122233; Email: chenxz@zjcc.org.cn \\ (C) Ivyspring International Publisher. Reproduction is permitted for personal, noncommercial use, provided that the article is in whole, unmodified, and properly cited. See \\ http://ivyspring.com/terms for terms and conditions.
}

Received: 2016.05.18; Accepted: 2016.08.08; Published: 2016.10.11

\begin{abstract}
This study aims to investigate the prognostic value of the C-reactive protein/albumin (CRP/ALB) ratio in nasopharyngeal carcinoma (NPC) in the intensity-modulated radiotherapy (IMRT) era. A total of 719 patients with NPC treated between January 2007 and December 2012 were retrospectively reviewed. Serum albumin and CRP levels were measured before treatment. The associations between the CRP/ALB ratio and clinicopathological parameters were analyzed. Multivariate analyses using the Cox proportional hazards model were performed to identify significant prognostic factors associated with overall survival (OS). The prognostic value of the CRP/ALB ratio was determined using receiver operating characteristic (ROC) curve analysis. The optimal CRP/ALB ratio cutoff value was 0.141 . High CRP/ALB ratio was significantly associated with older age $(P<0.001)$, more advanced T category $(P<0.001)$ and advanced TNM stage $(P=$ 0.024). Patients with an elevated CRP/ALB ratio $(\geq 0.141)$ had poorer OS than those with a CRPIALB ratio < 0.141 (5-year OS rates: $91.9 \%$ vs. $78.1 \% ; P<0.001)$. Multivariate analysis suggested clinical T category [hazard ratio (HR) 2.284; 95\% confidence interval (Cl), 1.429-3.652; $P=0.001$ ]; clinical $\mathrm{N}$ category (HR 1.575; $95 \% \mathrm{Cl}, 1.007-2.464 ; P=0.047)$ and CRP/ALB ratio (HR 2.173; 95\% Cl, 1.128-3.059; $P=0.015$ ) were independently associated with $\mathrm{OS}$. In conclusion, pretreatment CRP/ALB ratio is an objective biomarker with significant prognostic value for OS in NPC. The CRP/ALB ratio can enhance conventional TNM staging to stratify patients and may help facilitate individualized treatment of high-risk cases.
\end{abstract}

Key words: C-reactive protein/albumin ratio, nasopharyngeal carcinoma, overall survival, prognostic factor.

\section{Introduction}

Nasopharyngeal carcinoma (NPC) is endemic in Southern China, with an incidence of approximately 20-30 per 100000 [1, 2]. Radiotherapy is the mainstay of treatment for NPC [3]. The wide-spread adoption of intensity-modulated radiotherapy (IMRT) has revolutionized the management of NPC [1, 4-6].
However, vast inter-individual differences in treatment outcomes are observed among patients with same TNM (tumor-node-metastasis) stage of NPC. One possible explanation is that the TNM staging system does not accurately reflect intrinsic biological heterogeneity. Therefore, identification of 
objective and well-validated prognostic biomarkers may the complement TNM staging system and improve current treatment strategies for patients with NPC.

Accumulating evidence indicates that systemic inflammation plays important roles in the progression and prognosis of a variety of malignancies [7]. Recently, inflammation-based prognostic scores, including the modified Glasgow Prognostic Score (mGPS), Glasgow Prognostic Score (GPS), platelet to lymphocyte ratio (PLR) and neutrophil lymphocyte ratio (NLR), have been reported to have prognostic value in NPC [8-10].

Recently, a new novel inflammation-based prognostic score, the C-reactive protein albumin (CRP/ALB) ratio, has been revealed to have outstanding prognostic value in comparison with other inflammation-based prognostic scores in cancers, including hepatocellular carcinoma and esophageal cancer [11, 12]. However, to the best of our knowledge, the prognostic value of the CRP/ALB ratio has not yet been assessed in patients with NPC. Therefore, we conducted a large-sample retrospective study to evaluate the prognostic value of the CRP/ALB ratio in patients with NPC treated using IMRT. Moreover, we also investigated the association between the CRP/ALB ratio and other relevant clinicopathological factors in NPC.

\section{Materials and methods}

\section{Patient characteristics}

The clinical characteristics of NPC patients treated at Zhejiang Provincial Cancer Hospital, between January 2007 and December 2012 were reviewed retrospectively using medical records. This study was approved by the institutional ethical review board of Zhejiang Provincial Cancer Hospital. The inclusion criteria were as follows: (1) histologically-proven NPC; (2) no history of malignant disease; (3) no evidence of distant metastasis; and (4) no clinical evidence of infection or inflammatory conditions. A total of 719 NPC patients were included in the final evaluation.

Routine pre-treatment workup included a complete history, physical examination, hematology and biochemistry analyses, endoscopic examination of the nasopharynx, magnetic resonance imaging (MRI) of the nasopharynx and neck, chest radiography, abdominal ultrasonography, whole body bone scan. All patients were restaged according to the 7th edition of the American Joint Commission on Cancer (AJCC) staging system [13]. For evaluation of C-reactive protein (CRP) and serum albumin levels, the blood test results performed prior to initial treatment were used. The CRP/ALB ratio was calculated by dividing the serum CRP level by the serum albumin level [14].

\section{Treatment and follow-up}

All patients received IMRT combined with or without concurrent chemotherapy. The target volumes were delineated according to the institutional treatment protocol, which was based on International Commission on Radiation Units and Measurements reports (ICRU) 50 and 62. The prescribed radiation dose-range was a total dose of 69-69.9 Gy (30-33 fractions) to the primary gross tumor volume (GTV), 67.5-69.9 Gy to the positive lymph nodes (GTVnd), 60 Gy to the PTV of CTV-1 and 54 Gy to the PTV of CTV-2. All patients were treated with daily fractions over five days per week using a linear accelerator (6-8 MV).

According to institutional guidelines [6], a total of 698 patients $(97.1 \%)$ were treated with concurrent chemoradiotherapy and the remaining 21 patients received radiotherapy alone. In all, 649 patients $(90.3 \%)$ received induction chemotherapy and adjuvant chemotherapy was administered to 183 patients (25.5\%). Induction or adjuvant chemotherapy consisted of cisplatin with 5-fluorouracil or taxol every three weeks for two to three cycles. The concurrent chemotherapy regimen included cisplatin $\left(80 \mathrm{mg} / \mathrm{m}^{2}\right.$ intravenously in three days) and was administered every three weeks for two cycles.

Patients were regularly followed-up in our hospital. A physical examination, nasopharyngeal and neck MRI, nasopharyngoscopy, blood test, chest X-ray and abdominal sonogram were performed routinely every three months in the first three years, and every six months in the fourth to fifth years. The cutoff date of last follow-up was December 31st, 2014; the median follow-up duration was 48 months (range, 3-89 months).

\section{Statistical analysis}

Comparisons between groups were performed using the Mann-Whitney U test and Student's $t$-test for continuous variables and the Chi-square test for categorical variables.

The primary endpoint was overall survival (OS), which was calculated as the time from the first day after completion of radiotherapy to the date of death or censorship during follow-up. Receiver operating characteristic (ROC) curves were generated to select the optimal cutoff point with the highest Youden's index for OS. Survival curves were plotted using the Kaplan-Meier method and the log-rank test was used to assess the differences between survival rates. The Cox proportional hazards model was performed to 
analyze prognostic factors associated with OS. A two-tailed $P$ value $<0.05$ was considered statistically significant. All statistical analyses were performed using SPSS v18.0 (SPSS, Chicago, IL, USA).

\section{Results}

\section{Patient characteristics}

A total of 719 patients were included in this study, with a predominance of males (68.8\%). The median age of the cohort was 48 (range, 14-81) years. According to the 7th AJCC TNM staging system, $14 / 719(1.9 \%)$ patients were classified as stage I, 98 $(13.6 \%)$ as stage II, $381(53.0 \%)$ as stage III, and 226 $(31.4 \%)$ as stage IV. Six hundred and ninety-eight patients (97.1\%) received concurrent chemoradiotherapy and $21 / 719(2.9 \%)$ patients under went radiotherapy alone.

The median CRP/ALB ratio was 0.138 (range, 0.010-1.932). ROC curves were constructed for OS, and the AUC was used to assess the discriminative ability of the CRP/ALB ratio (Fig. 1). The optimal CRP/ALB ratio cutoff value for OS was 0.141 , with a sensitivity and specificity of $86.4 \%$ and $57.5 \%$, respectively. The patients were divided into two groups according to this cutoff value $(<0.141, n=378$; $\geq 0.141, n=341)$.

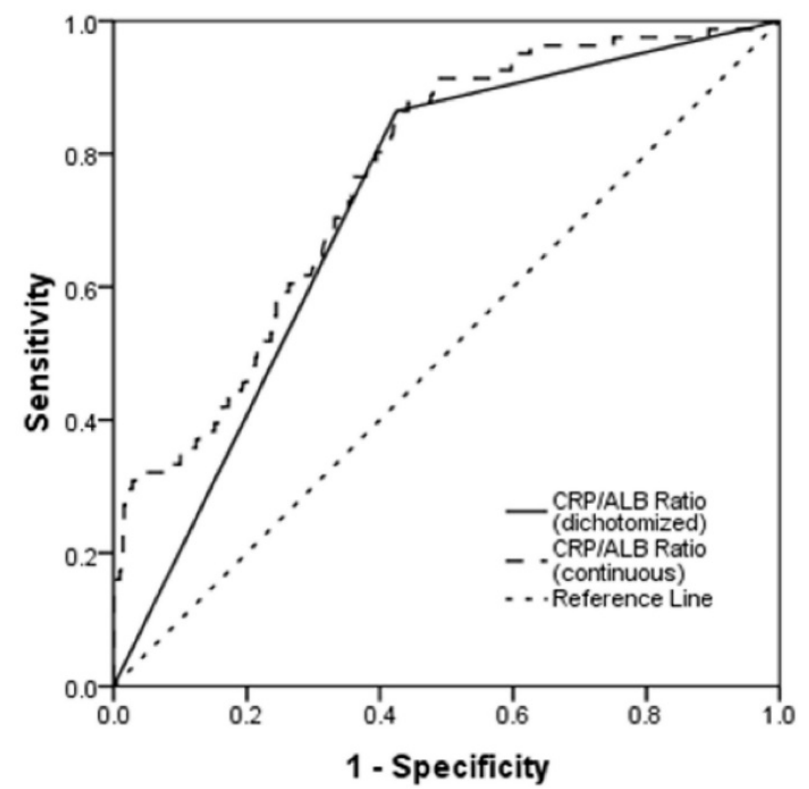

Fig. 1. Receiver operating curve (ROC) analysis of the CRP/ALB ratio for survival status among the 719 patients with NPC. The optimal cutoff value was 0.141 (sensitivity: $86.4 \%$; specificity: $57.5 \%$ ) according to the ROC analysis.

Table 1 presents the associations of the $\mathrm{CRP} / \mathrm{ALB}$ ratio with clinicopathological characteristics. A high CRP/ALB ratio was significantly associated with older age $(P<0.001)$, more advanced $\mathrm{T}$ category $(P<0.001)$ and more advanced TNM stage $(P=0.024)$. No significant associations were observed between the CRP/ALB ratio and sex, Karnofsky performance score (KPS), histological type, $\mathrm{N}$ category, and induction chemotherapy or concurrent chemotherapy.

The correlations between the CRP/ALB ratio and continuous serum variables are shown in Table 2. Patients with a higher CRP/ALB ratio $(\geq 0.141)$ had higher white blood cell counts $(P=0.018)$, a higher percentage of lymphomonocytes $(P=0.027)$ and a higher percentage of natural killer cells $(P=0.028)$ in comparison with patients who had a CRP/ALB ratio $<0.141$. There were no significant differences in neutrophil granulocytes, lymphocytes, hemoglobin, platelets or serum lactic dehydrogenase between the two groups.

Table 1. Baseline characteristics of the study population of 719 patients with NPC.

\begin{tabular}{|c|c|c|c|c|}
\hline \multirow[t]{2}{*}{ Characteristic } & \multirow[t]{2}{*}{ Total (\%) } & \multicolumn{2}{|c|}{ CRP/ALB ratio } & \multirow[t]{2}{*}{$P$} \\
\hline & & $<0.141$ & $\geq 0.141$ & \\
\hline Total & 719 & 378 & 341 & \\
\hline Age (years) & & & & $<0.001$ \\
\hline$\leq 50$ & $382(53.1)$ & $232(61.4)$ & $150(44.0)$ & \\
\hline$>50$ & 337 (46.9) & $146(38.6)$ & $191(56.0)$ & \\
\hline Sex & & & & 0.334 \\
\hline Male & $495(68.8)$ & $254(67.2)$ & 241 (70.7) & \\
\hline Female & $224(31.2)$ & $124(32.8)$ & $100(29.3)$ & \\
\hline KPS & & & & 0.420 \\
\hline$\geq 90$ & 694 & 367 (97.1) & 327 (95.9) & \\
\hline$<90$ & 25 & $11(2.9)$ & $14(4.1)$ & \\
\hline Histological type & & & & 0.884 \\
\hline WHO type I & $50(6.9)$ & $27(7.1)$ & $23(6.7)$ & \\
\hline WHO type II/III & $619(93.1)$ & 351 (92.9) & $318(93.4)$ & \\
\hline $\mathrm{T}$ category & & & & $<0.001$ \\
\hline $\mathrm{T} 1$ & $73(10.2)$ & $48(12.7)$ & $25(7.3)$ & \\
\hline $\mathrm{T} 2$ & 107 (14.9) & $64(16.9)$ & $43(12.7)$ & \\
\hline $\mathrm{T} 3$ & $353(49.1)$ & $192(50.8)$ & $161(47.2)$ & \\
\hline $\mathrm{T} 4$ & $186(25.9)$ & 74 (19.6) & $112(32.8)$ & \\
\hline $\mathrm{N}$ category & & & & 0.842 \\
\hline No & $80(11.1)$ & $42(11.1)$ & 38 (11.1) & \\
\hline N1 & $368(51.2)$ & 195 (51.6) & $173(50.7)$ & \\
\hline N2 & $213(29.6)$ & $110(29.1)$ & 113 (29.9) & \\
\hline N3 & $58(8.1)$ & $31(8.2)$ & $27(7.9)$ & \\
\hline TNM stage & & & & 0.024 \\
\hline I-II & 112 (15.6) & $70(18.5)$ & $42(12.3)$ & \\
\hline III-IV & $607(84.4)$ & $308(81.5)$ & 299 (87.7) & \\
\hline $\begin{array}{l}\text { Induction } \\
\text { chemotherapy }\end{array}$ & & & & 0.451 \\
\hline Yes & 649 (90.3) & $338(89.4)$ & 311 (91.2) & \\
\hline No & $70(9.7)$ & $40(10.6)$ & $30(8.8)$ & \\
\hline $\begin{array}{l}\text { Concurrent } \\
\text { chemotherapy }\end{array}$ & & & & 0.664 \\
\hline Yes & 698 (97.1) & $368(97.4)$ & $330(96.8)$ & \\
\hline No & $21(2.9)$ & $10(2.6)$ & $11(3.2)$ & \\
\hline
\end{tabular}

\section{Survival analysis}

During follow-up, a total of $81(11.3 \%)$ patients died of NPC. Loco-regional failure was detected in 76 patients $(10.6 \%), 60(8.3 \%)$ patients developed distant metastases, and 14 (1.9\%) patients developed both 
local and distant failures. The median time of OS in the whole cohort was 47.0 months. The interquartiles (P25; P75) of OS time was 34.0 months and 56.0 months respectively. In patients with CRP/ALB ratio $<0.141$, the median time of OS was 48.0 months and the interquartiles (P25; P75) was 40.0 months, 59.0 months, respectively. In patients with CRP/ALB ratio $\geq 0.141$, the median time of OS was 41.0 months and the interquartiles (P25; P75) was 34.0 months, 50.0 months, respectively. For the entire cohort, the 5-year OS, distant metastasis-free survival (DMFS) and local recurrence-free survival (LRFS) rates were 85.8\%, $89.4 \%$ and $86.7 \%$, respectively.

Table 3 summarizes the results of univariate analysis. Univariate analysis revealed that age $(P=$ $0.011)$, clinical $\mathrm{T}$ category $(P<0.001)$, clinical $\mathrm{N}$ category $(P=0.025)$ and the $\mathrm{CRP} / \mathrm{ALB}$ ratio $(P<$ $0.001)$ were significantly associated with OS. The 1-, 3 -, and 5-year OS rates for patients with a CRP/ALB ratio $<0.141$ were $98.7,94.5$, and $91.9 \%$, respectively. These OS rates were significantly better than those of patients with a CRP/ALB ratio $\geq 0.141$, which were $98.2,89.0$, and $78.1 \%$, respectively $(P<0.001$; Fig. 2$)$.

The variables that were significant in univariate analysis were subjected to multivariate analysis to identify independent prognostic indicators for OS. Multivariate analysis suggested that clinical $\mathrm{T}$ category [hazard ratio (HR) 2.284; 95\% confidence interval [CI], 1.429-3.652; $P=0.001]$; clinical $\mathrm{N}$ category (HR 1.575; 95\% CI, 1.007-2.464; $P=0.047$ ) and the CRP/ALB ratio (HR 2.173; 95\% CI, 1.128-3.059; $P=0.015)$ were independent indicators affecting OS (Table 3).

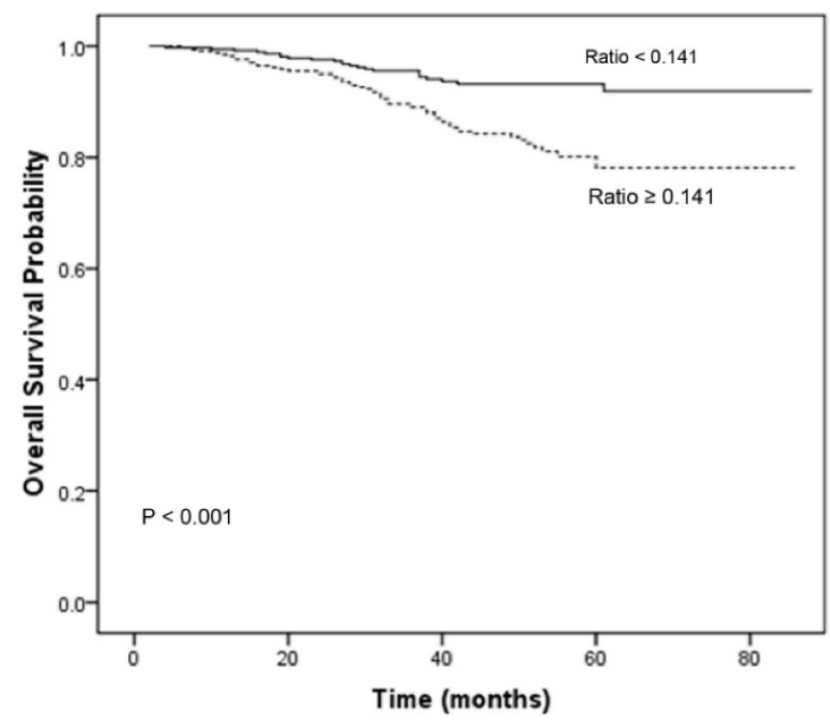

Fig. 2. Overall survival curves for the 719 patients with NPC stratified by the CRP/ALB ratio $(P<0.001)$. CRPIALB C-reactive protein/albumin.
Table 2. Relationship between the CRP/ALB ratio and the serum continuous variables of the 719 patients with NPC.

\begin{tabular}{llll}
\hline Variable & CRP/ALB & CRP/ALB & $P$ \\
& $<0.141$ & $\geq 0.141$ & \\
\hline White blood cell (count/L) & $6.5 \pm 1.1$ & $7.2 \pm 1.3$ & 0.018 \\
Neutrophil granulocyte (count/L) & $3.93 \pm 1.31$ & $4.04 \pm 1.62$ & 0.292 \\
Lymphomonocytes (\%) & $48.5 \pm 15$ & $51.4 \pm 19$ & 0.027 \\
Lymphocytes (count/L) & $1.86 \pm 0.57$ & $1.78 \pm 0.59$ & 0.116 \\
Natural killer cells (\%) & $23.9 \pm 10.2$ & $25.6 \pm 10.7$ & 0.028 \\
Hemoglobin (g/dL) & $132.3 \pm 10.5$ & $133.1 \pm 11.3$ & 0.132 \\
Platelets (count/L) & $258.2 \pm 43.7$ & $256.8 \pm 42.8$ & 0.258 \\
Serum LDH (IU/L) & $174.4 \pm 40.8$ & $178.7 \pm 43.3$ & 0.168 \\
Total bilirubin (mg/dL) & $12.3 \pm 4.3$ & $12.1 \pm 4.1$ & 0.835 \\
Direct bilirubin (mg/dL) & $8.8 \pm 3.8$ & $8.7 \pm 3.5$ & 0.476 \\
Indirect bilirubin (mg/dL) & $3.5 \pm 1.4$ & $3.4 \pm 1.6$ & 0.758 \\
Aspartate aminotransferase (AST, U/L) & $24.2 \pm 6.9$ & $23.4 \pm 7.5$ & 0.321 \\
Alanine aminotransferase (ALT, U/L) & $20.4 \pm 4.6$ & $20.2 \pm 3.9$ & 0.435 \\
\hline
\end{tabular}

Continuous data are presented as mean \pm standard deviation.

Table 3. Univariate and multivariate analysis for overall survival (OS) for the 719 patients with NPC.

\begin{tabular}{|c|c|c|c|c|c|c|}
\hline \multirow[t]{2}{*}{ Variables } & \multirow[t]{2}{*}{ No. } & \multicolumn{2}{|c|}{ Univariate analysis } & \multicolumn{3}{|c|}{ Multivariate analysis } \\
\hline & & 5-year OS & $P$ & $\mathrm{HR}$ & $95 \% \mathrm{CI}$ & $P$ \\
\hline Sex & & & 0.080 & 0.746 & $0.440-1.263$ & 0.275 \\
\hline Male & 495 & $82.7 \%$ & & & & \\
\hline Female & 224 & $90.8 \%$ & & & & \\
\hline Age (years) & & & 0.011 & 1.434 & $0.924-2.224$ & 0.108 \\
\hline$<50$ & 382 & $91.7 \%$ & & & & \\
\hline$\geq 50$ & 337 & $83.7 \%$ & & & & \\
\hline $\mathrm{LDH}(\mathrm{IU} / \mathrm{L})$ & & & 0.056 & 1.248 & $0.875-2.135$ & 0.105 \\
\hline$<187$ & 500 & $88.1 \%$ & & & & \\
\hline$\geq 187$ & 218 & $79.9 \%$ & & & & \\
\hline Neoadjuvant CT & & & 0.496 & & & \\
\hline Yes & 649 & $81.1 \%$ & & & & \\
\hline No & 70 & $85.7 \%$ & & & & \\
\hline Therapy & & & 0.686 & & & \\
\hline CCRT & 698 & $84.7 \%$ & & & & \\
\hline RT alone & 21 & $85.8 \%$ & & & & \\
\hline T category & & & $<0.001$ & 2.284 & $1.429-3.652$ & 0.001 \\
\hline T 1-3 & 533 & $87.9 \%$ & & & & \\
\hline $\mathrm{T} 4$ & 186 & $77.1 \%$ & & & & \\
\hline $\mathrm{N}$ category & & & 0.025 & 1.575 & $1.007-2.464$ & 0.047 \\
\hline N $0-1$ & 446 & $87.7 \%$ & & & & \\
\hline N 2-3 & 273 & $82.2 \%$ & & & & \\
\hline CRP (mg/L) & & & 0.258 & & & \\
\hline$<10$ & 558 & $85.6 \%$ & & & & \\
\hline$\geq 10$ & 161 & $83.8 \%$ & & & & \\
\hline Albumin (g/L) & & & 0.157 & & & \\
\hline$\geq 43$ & 443 & $87.5 \%$ & & & & \\
\hline$<43$ & 275 & $82.4 \%$ & & & & \\
\hline $\mathrm{CRP} / \mathrm{ALB}$ ratio & & & $<0.001$ & 2.173 & $1.128-3.059$ & 0.015 \\
\hline$<0.141$ & 378 & $91.9 \%$ & & & & \\
\hline$\geq 0.141$ & 341 & $78.1 \%$ & & & & \\
\hline
\end{tabular}

To further analyze the prognostic value of CRP/ALB ratio in NPC patients, patients were then categorized into 4 subgroups (Quartile 1-4) according to the quartiles of CRP/ALB ratio $(<0.092$; $0.092-0.138 ; 0.138-0.178 ; \geq 0.178)$. The relationships between different categories of CRP/ALB ratio (Quartile 1-4) and TNM stage were shown in the 
Table S1. There was statistical significance in the distribution of TNM stage among the different categories of CRP/ALB ratio $(P<0.001$; chi-square test). In univariate analyses, the category (Quartile 1-4) was statistically significantly associated with OS $(P<0.001$, Figure 3$)$. In the multivariate analyses by the Cox proportional hazards model, the category (Quartile 1-4) was an independent factor for OS ( $P=$ 0.003). Compared with the Quartile 1 which served as a reference group, the HRs $(95 \% \mathrm{CI})$ were 3.622 (0.992-13.223) for Quartile 2, 8.187 (2.471-27.125) for Quartile 3 and 10.927 (3.338-35.773) for Quartile 4 after adjusting for the variables which were significantly associated with OS in the univariate analyses, including sex, age, $\mathrm{LDH}, \mathrm{T}$ classification and $\mathrm{N}$ classification.

In patients with stage I-II (112 patients), patients with a lower CRP/ALB ratio had a better OS than those with higher CRP/ALB ratio, but there was no statistical significance (5-year OS rates: $91.6 \%$ vs. $85.5 \% ; P=0.301$; Figure S1A). In patients with stage III-IV (607 patients), patients with a lower CRP/ALB ratio had a significant better OS than those with higher CRP/ALB ratio (5-year OS rates: $86.6 \%$ vs. $77.0 \% ; P<0.001$; Figure S1B).

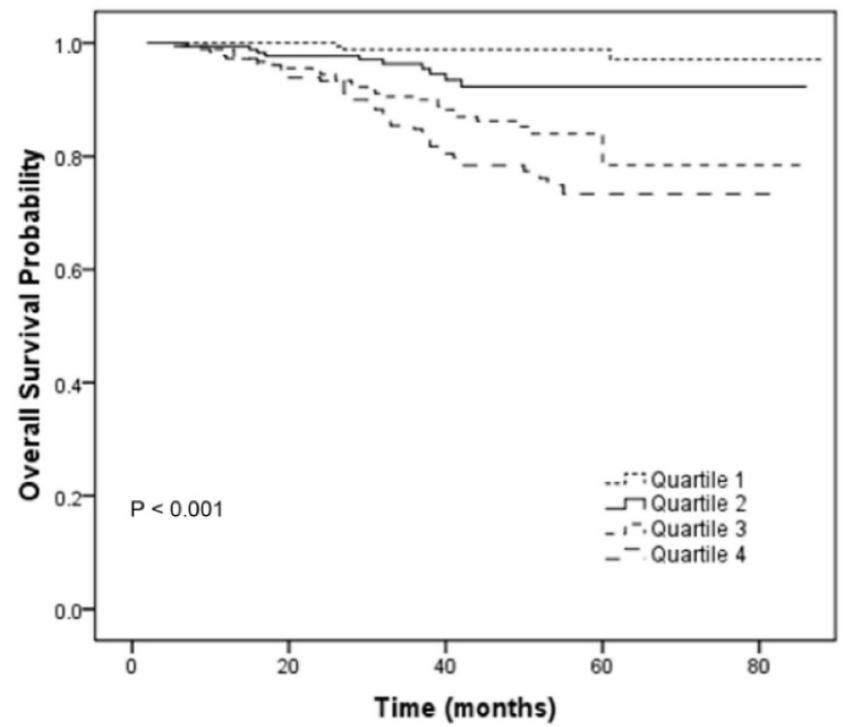

Fig. 3. Overall survival curves for the 719 patients with NPC stratified by 4 categories of CRPIALB ratio (Quartile 1-4; $P<0.001$ ).

\section{Discussion}

In this study, we retrospectively analyzed 719 eligible patients and found that the CRP/ALB ratio was an independent prognostic factor for OS in patients with NPC treated with IMRT. To our best knowledge, this is the first study to investigate the prognostic value of the CRP/ALB ratio in NPC patients receiving IMRT.
Accumulating evidence has clarified the important role of systemic inflammation in the prognosis of patients with cancer [15]. In addition, inflammation can be promoted by inflammatory cytokines produced by cancer cells in the tumor microenvironment [16]. Moreover, inflammatory cells may promote cancer metastasis by adhering molecules and chemokines [17].

Several inflammation-based prognostic scores (GPS, mGPS etc.) which combine serum CRP and albumin levels have been explored as prognostic factors for the outcomes of patients with various types of cancer, especially cancers of the digestive tract. However, in contrast to other malignancies, the majority of patients with NPC have good performance status and nutritional status at diagnosis. In this study, only four patients $(0.5 \%)$ had hypoalbuminemia at diagnosis. The fact that few patients with NPC have hypoalbuminemia before treatment limits the application of other prognostic scores that classify patients into categories based on the presence or absence of hypoalbuminemia, such as the GPS and mGPS. Conversely, the CRP/ALB ratio is a continuous variable that not only reflects systemic inflammation and nutritional status, but is also calculated using two continuous variables (CRP level and serum albumin level), which could reduce the potential for underestimation or overestimation and stratify patients to predict survival more accurately. The CRP/ALB ratio was originally developed by Fairclough et al. to predict the patient outcome [14]. The CRP/ALB ratio has been identified as an independent prognostic factor in several malignancies, including hepatocellular carcinoma [11], esophageal cancer [12], pancreatic cancer [18] and small cell lung cancer [19]. Furthermore, Ranzani et al. revealed the CRP/ALB ratio was more accurate than CRP level alone for predicting mortality in patients with sepsis [14]. In this study, the optimal CRP/ALB ratio cutoff value of 0.141 was applied to predict OS, and we observed that the CRP/ALB ratio was significantly associated with OS in patients with NPC who received IMRT.

The CRP/ALB ratio indicates the interactive relationship between systemic inflammatory responses and dystrophia. The mechanisms that regulate the association between the CRP/ALB ratio and the survival of patients with cancer are not yet completely elucidated. Several explanations have been proposed. Firstly, an elevated CRP level generally suggests the presence of a systemic inflammatory response [15]. Tumor invasion and tumor cell necrosis could promote an inflammatory response in itself [20]. Marsik et al. indicated that 10 -year all-cause mortality of patients with a higher 
CRP level (> $80 \mathrm{mg} / \mathrm{L})$ was almost $30 \%$ and that a highly elevated CRP level was associated with significantly higher mortality due to cancer, non-cancer or all-cause vascular diseases after retrospective analysis of 274,515 patients [15]. In addition, most patients with NPC are infected with the Epstein-Barr virus (EBV), which has been confirmed as a prognostic factor in NPC [21, 22]. Infection by EBV could stimulate the production of inflammatory cytokines including interleukin (IL)-1, IL-6, IL-8, vascular endothelial growth factor (VEGF) and tumor necrosis factor (TNF)- $\alpha$, which could induce CRP synthesis in the liver and reduces the levels of albumin produced by hepatic cells [23, 24]. Furthermore, tumor-associated leukocytes and/or cancer cells could also release these aforementioned inflammatory cytokines, which in turn could promote tumor invasion and metastases, subvert the host immune response and led to increased resistance to chemotherapy based on cytotoxic drugs [16, 25-27].

It is noteworthy that these findings may have important implications for individualized treatment and surveillance of patients with NPC. Patients with an elevated CRP/ALB ratio may require closer follow-up and may also benefit from nutritional support and anti-inflammatory therapy [28, 29]. Furthermore, Mocellin et al. demonstrated that supplementation with encapsulated fish oil (providing $600 \mathrm{mg} /$ day of eicosapentaenoic acid and docosahexaenoic acid) could decrease the CRP/ALB ratio and potentially reduce weight loss in patients with colorectal cancer [30]. Although the CRP/ALB ratio appears to accurately stratify patients with NPC on the basis of OS and has the potential to inform treatment selection, the CRP/ALB ratio should be combined with the standard TNM staging system instead of replacing it.

This study has several potential limitations. This analysis was inevitably biased by its retrospective nature and the fact it was a single-institution study. It is also worth noting that patients with NPC may have co-morbidities that could raise the serum CRP level and affect albumin and other biochemical parameters. In addition, data on plasma EBV DNA concentrations was not assessed in this study because EBV DNA copy number was not routinely determined for this cohort of patients. However, this study was based on a large, homogeneous population of patients with NPC who received IMRT.

In conclusion, this study demonstrates that the $\mathrm{CRP} / \mathrm{ALB}$ ratio is an independent prognostic factor for overall survival patients with NPC who receive IMRT. As a readily-available and easily-measurable biomarker, combination of the CRP/ALB ratio with the conventional TNM staging system should be explored to predict survival more accurately in patients with NPC receiving IMRT.

\section{Supplementary Material}

Supplementary table and figure. http://www.jcancer.org/v07p2005s1.pdf

\section{Acknowledgement}

This work was supported by grants from the Natural Science Foundation of China (No. 81372437) and Natural Science Foundation of China (No. 81502647).

\section{Competing Interests}

The authors have declared that no competing interest exists.

\section{References}

1. Wei WI, Sham JS. Nasopharyngeal carcinoma. Lancet. 2005;365:2041-54.

2. Chan AT. Nasopharyngeal carcinoma. Ann Oncol. 2010;21 (Suppl 7):vii308-12.

3. Chan AT, Teo PM, Johnson PJ. Nasopharyngeal carcinoma. Ann Oncol. 2002;13:1007-15.

4. Wolden SL, Chen WC, Pfister DG, et al. Intensity-modulated radiation therapy (IMRT) for nasopharynx cancer: update of the Memorial Sloan-Kettering experience. Int J Radiat Oncol Biol Phys. 2006;64:57-62.

5. Wee J, Tan EH, Tai BC, et al. Randomized trial of radiotherapy versus concurrent chemoradiotherapy followed by adjuvant chemotherapy in patients with American Joint Committee on Cancer/International Union against cancer stage III and IV nasopharyngeal cancer of the endemic variety. J Clin Oncol. 2005;23:6730-8.

6. Jiang $\mathrm{F}$, Jin $\mathrm{T}$, Feng $\mathrm{XL}$, et al. Long-term outcomes and failure patterns of patients with nasopharyngeal carcinoma staged by magnetic resonance imaging in intensity-modulated radiotherapy era: The Zhejiang Cancer Hospital's experience. J Cancer Res Ther. 2015;11 (Suppl 2):179-84.

7. Mantovani A. Cancer: Inflaming metastasis. Nature. 2009;457:36-7.

8. Sun W, Zhang L, Luo M, et al. Pretreatment hematologic markers as prognostic factors in patients with nasopharyngeal carcinoma: Neutrophil-lymphocyte ratio and platelet-lymphocyte ratio. Head Neck. 2016;38 (Suppl 1):1332-40.

9. Chen C, Sun P, Dai QS, et al. The Glasgow Prognostic Score predicts poor survival in cisplatin-based treated patients with metastatic nasopharyngeal carcinoma. PloS One. 2014;9:e112581.

10. Chen $\mathrm{Q}$, Yang $\mathrm{LX}, \mathrm{Li} \mathrm{XD}$, et al. The elevated preoperative neutrophil-to-lymphocyte ratio predicts poor prognosis in intrahepatic cholangiocarcinoma patients undergoing hepatectomy. Tumour Biol. 2015;36:5283-9.

11. Kinoshita A, Onoda $\mathrm{H}$, Imai N, et al. The C-reactive protein/albumin ratio, a novel inflammation-based prognostic score, predicts outcomes in patients with hepatocellular carcinoma. Annals of surgical oncology. 2015;22:803-10.

12. $\mathrm{Xu} \mathrm{XL}, \mathrm{Yu} \mathrm{HQ}, \mathrm{Hu} \mathrm{W}$, et al. A Novel Inflammation-Based Prognostic Score, the C-Reactive Protein/Albumin Ratio Predicts the Prognosis of Patients with Operable Esophageal Squamous Cell Carcinoma. PloS one. 2015;10:e0138657.

13. Edge SB, Compton CC. The American Joint Committee on Cancer: the 7th edition of the AJCC cancer staging manual and the future of TNM. Annals of surgical oncology. 2010;17:1471-4.

14. Fairclough E, Cairns E, Hamilton J, et al. Evaluation of a modified early warning system for acute medical admissions and comparison with C-reactive protein/albumin ratio as a predictor of patient outcome. Clin Med (Lond). 2009;9:30-3.

15. Marsik C, Kazemi-Shirazi L, Schickbauer T, et al. C-reactive protein and all-cause mortality in a large hospital-based cohort. Clinical chemistry. 2008;54:343-9.

16. Balkwill F, Mantovani A. Inflammation and cancer: back to Virchow? Lancet. 2001;357:539-45.

17. Grivennikov SI, Greten FR, Karin M. Immunity, inflammation, and cancer. Cell. 2010;140:883-99.

18. Haruki K, Shiba H, Shirai Y, et al. The C-reactive Protein to Albumin Ratio Predicts Long-Term Outcomes in Patients with Pancreatic Cancer After Pancreatic Resection. World journal of surgery. 2016.

19. Zhou T, Zhan J, Hong S, et al. Ratio of C-Reactive Protein/Albumin is An Inflammatory Prognostic Score for Predicting Overall Survival of Patients with Small-cell Lung Cancer. Scientific reports. 2015;5:10481.

20. Zhang $\mathrm{P}, \mathrm{Xi} \mathrm{M}, \mathrm{Li} \mathrm{QQ}$, et al. The modified glasgow prognostic score is an independent prognostic factor in patients with inoperable thoracic esophageal 
squamous cell carcinoma undergoing chemoradiotherapy. Journal of Cancer. 2014;5:689-95.

21. Senba M, Zhong XY, Senba MI, et al. EBV and nasopharyngeal carcinoma. Lancet. 1994;343:1104.

22. Twu CW, Wang WY, Liang WM, et al. Comparison of the prognostic impact of serum anti-EBV antibody and plasma EBV DNA assays in nasopharyngeal carcinoma. Int J Radiat Oncol Biol Phys. 2007;67:130-7.

23. Eliopoulos AG, Stack M, Dawson CW, et al. Epstein-Barr virus-encoded LMP1 and CD40 mediate IL-6 production in epithelial cells via an NF-kappaB pathway involving TNF receptor-associated factors. Oncogene. 1997;14:2899-916.

24. Pepys MB, Hirschfield GM. C-reactive protein: a critical update. J Clin Invest. 2003;111:1805-12.

25. Heikkila K, Ebrahim S, Lawlor DA. A systematic review of the association between circulating concentrations of $\mathrm{C}$ reactive protein and cancer. J Epidemiol Community Health. 2007;61:824-33.

26. Mantovani A, Allavena P, Sica A, et al. Cancer-related inflammation. Nature. 2008;454:436-44.

27. Elinav E, Nowarski R, Thaiss CA, et al. Inflammation-induced cancer: crosstalk between tumours, immune cells and microorganisms. Nat Rev Cancer. 2013;13:759-71.

28. Rizzo A, Cengel KA. Anti-inflammatory therapy for pancreatic cancer: a sorely needed advance in therapeutics. Cancer Biol Ther. 2008;7:1051-2.

29. Langman MJ, Cheng KK, Gilman EA, et al. Effect of anti-inflammatory drugs on overall risk of common cancer: case-control study in general practice research database. BMJ. 2000;320:1642-6.

30. Mocellin MC, Pastore e Silva Jde A, Camargo Cde Q, et al. Fish oil decreases C-reactive protein/albumin ratio improving nutritional prognosis and plasma fatty acid profile in colorectal cancer patients. Lipids. 2013;48:879-88. 